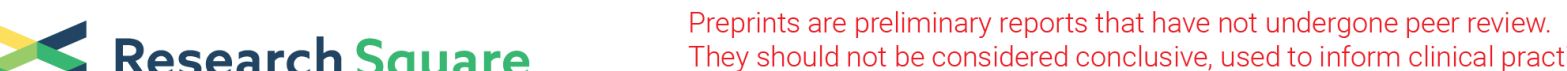 $\begin{array}{ll}\text { Research Square } & \text { They should not be considered conclusive, used to inform clinical practice, } \\ \text { or referenced by the media as validated information. }\end{array}$
}

\section{Estimating the Risk of Ulcerative Colitis-Associated Pyoderma Gangrenosum: A Population-Based Case- Control Study}

Khalaf Kridin ( $\nabla$ dr_kridin@hotmail.com )

Universitat zu Lubeck https://orcid.org/0000-0001-9971-9151

Giovanni Damiani

UH Cleveland Medical Center

Ralf J Ludwig

Universitat zu Lubeck

Dana Tzur Bitan

Ariel University

Arnon D. Cohen

Clalit Health Services

\section{Research}

Keywords: Pyoderma gangrenosum, Ulcerative colitis, Case-control study

Posted Date: May 4th, 2020

DOI: https://doi.org/10.21203/rs.3.rs-26104/v1

License: (우 This work is licensed under a Creative Commons Attribution 4.0 International License. Read Full License 


\section{Abstract}

\section{Background}

Ulcerative colitis (UC) is a well-known underlying comorbidity in pyoderma gangrenosum (PG). However, the relative risk conferred by UC for the subsequent development of $P G$ is yet to be explicated.

\section{Objective}

To estimate the magnitude of the association between UC and the subsequent occurrence of PG, thus enabling to assess the PG risk with UC.

\section{Methods}

A population-based case-control study was conducted comparing PG patients $(n=302)$ with age-, sex-and ethnicity-matched control subjects $(n=1,497)$ regarding the presence of UC. Logistic regression models were utilized for univariate and multivariate analyses.

\section{Results}

The prevalence of UC was greater in patients with PG than in control subjects ( $7.3 \%$ vs. $0.5 \%$, respectively; $P<0.001)$. A 15-fold increase in the odds of $P G$ with UC (OR, $14.6295 \% \mathrm{Cl}, 6.45-33.18)$ was observed. This association retained its statistical significance following a sensitivity analysis excluding UC cases diagnosed up to 2 years prior to PG (OR, 9.88; 95\% Cl, 3.91-24.97), and after adjusting for confounding factors (adjusted OR, 10.78; $95 \% \mathrm{Cl}, 4.55-25.52$ ). The median latency between the diagnosis of UC and the development of PG was 4.59 years. Patients with both PG and UC were younger and had a lower prevalence of smoking when compared to the remaining patients with PG.

\section{Conclusions}

UC increases the odds of developing PG by 15 -folds. Physicians managing patients with UC should be aware of this increased burden. Patients with UC may be advised to avoid additional precipitating factors of PG.

\section{Background}

Pyoderma gangrenosum $(\mathrm{PG})$ is a rare neutrophilic inflammatory dermatosis that typically presents with one or more painful ulcers with violaceous, undermined borders on the lower extremities. Less common morphological phenotypes include pustules or tender nodules on other anatomical locations[1]. Histologically, it is typified by a neutrophil-rich infiltrate. PG may be sporadic or present in syndromic forms, where it is regarded as a polygenic autoinflammatory disease[2, 3]. More than half of patients with sporadic PG have an underlying systemic disease, mainly inflammatory bowel disease (IBD), rheumatoid arthritis, and hematological malignancies[4]. 
Ulcerative colitis (UC) is a chronic idiopathic inflammatory bowel disorder manifesting with continuous mucosal inflammation extending from the rectum to the more proximal portions of the colon. The disease is characterized by a relapsing and remitting course imposing a high burden of morbidly and substantial disruption in quality of life[5]. While the incidence and prevalence of UC demonstrate a decreasing or stabilizing trend in Europe and North America during the past three decades, its incidence has been increasing in newly industrialized countries in Asia, Africa, and South America[6]. UC may be complicated with extraintestinal manifestation involving musculoskeletal, cutaneous, ocular, and hepatobiliary conditions[7].

UC was long considered as one of the leading underlying comorbidity among patients with PG. The pooled prevalence of UC among patients with PG had been estimated at $11.5 \%(95 \% \mathrm{Cl}, 7.2-16.6 ; \mathrm{I} 2=83.7 \%)$ in a recent meta-analysis synthesizing data across 17 cohorts of patients with PG[4]. Correspondingly, the incidence of PG among patients with IBD was estimated at $0.6 \%$ in a meta-analysis summarizing cohorts of IBD patients[8]. Nonetheless, patients with PG were not compared with matched controls with regard to the presence of UC. Thus, the relative risk of developing PG after the occurrence of UC is yet to be outlined.

The aim of the current study is to quantify the association between previous UC and the later occurrence of $P G$ in a population-based case-control study. Additionally, we aimed to evaluate whether patients with comorbid PG and UC have distinct epidemiological features distinguishing them from other patients with PG.

\section{Results}

The study population comprised of 302 patients with PG and 1497 matched control individuals. The mean (SD) age at the diagnosis of patients and enrollment of control individuals was 54.0 (20.8) years. Onehundred-twenty-seven (42.1\%) patients with PG were males, and 255 (84.4\%) were of Jewish ancestry. The sex distribution and ethnic background were comparable between the two groups (Table 1). Moreover, the average BMI and the prevalence of smoking were not significantly different in the two groups (Table 1). The mean (SD) Charlson comorbidity score was higher in case than in control individuals (2.3 [2.7] vs. 1.3 [1.8], respectively; $P<0.001)$. A larger percentage of cases suffered from severe comorbidity relative to controls $(37.4 \%$ vs. $19.2 \%$, respectively; $P<0.001)$. Characteristics of case and control participants are demonstrated in Table 1. 
Table 1

Descriptive characteristics of the study population

\begin{tabular}{|c|c|c|c|}
\hline Characteristic & Patients with pyoderma gangrenosum $(\mathrm{N}=302)$ & $\begin{array}{l}\text { Controls } \\
(N=1,497)\end{array}$ & $P$ value \\
\hline \multicolumn{4}{|l|}{ Age, years } \\
\hline Mean \pm SD & $54.0 \pm 20.8$ & $54.0 \pm 20.8$ & \multirow[t]{2}{*}{1.000} \\
\hline Median (range) & $55.8(0.2-95.1)$ & $55.9(0.2-95.6)$ & \\
\hline Male sex, N (\%) & $157(57.9 \%)$ & $629(58.0 \%)$ & 0.974 \\
\hline \multicolumn{4}{|l|}{ Ethnicity, N (\%) } \\
\hline Jews & $255(84.4 \%)$ & $1264(84.4 \%)$ & \multirow[t]{2}{*}{1.000} \\
\hline Arabs & $47(15.6 \%)$ & $233(15.6 \%)$ & \\
\hline \multicolumn{4}{|l|}{$\mathrm{BMI}, \mathrm{mg} / \mathrm{kg}^{2}$} \\
\hline Mean \pm SD & $28.0 \pm 6.3$ & $27.8 \pm 6.2$ & 0.614 \\
\hline Smoking, N (\%) & $115(38.1 \%)$ & $521(34.8 \%)$ & 0.274 \\
\hline \multicolumn{4}{|c|}{ Charlson comorbidity score } \\
\hline Mean score \pm SD & $2.3 \pm 2.7$ & $1.3 \pm 1.8$ & $<0.001$ \\
\hline None $(0)$ & $111(36.8 \%)$ & $777(51.9 \%)$ & $<0.001$ \\
\hline Moderate (1-2) & $78(25.8 \%)$ & $432(28.9 \%)$ & 0.276 \\
\hline Severe $(\geq 3)$ & $113(37.4 \%)$ & $288(19.2 \%)$ & $<0.001$ \\
\hline
\end{tabular}

The prevalence of UC was greater among patients with PG than among control subjects $(7.3 \%$ vs. $0.5 \%$, respectively; $P<0.001)$. A 15 -fold increase in the odds of $P G$ with UC was observed $(O R, 14.62 ; 95 \% \mathrm{Cl}$, 6.45-33.18). When stratified by age, sex, and ethnicity, the association between PG and UC was more prominent among younger (< 54 years; OR, 84.00; 95\% Cl, 10.99-641.61), female (OR, 31.02; 95\% Cl, 8.99107.10), and $A r a b(O R, 21.67 ; 95 \% \mathrm{Cl}, 2.37-198.63)$ patients, respectively. Intriguingly, the association was stronger among non-smokers (OR, 21.96; 95\% Cl, 8.09-59.62; Table 2). 
Table 2

The risk of pyoderma gangrenosum in patients with a preceding diagnosis of ulcerative colitis stratified by age, sex, and ethnicity

\begin{tabular}{|c|c|c|c|c|c|}
\hline Subgroup & Number & $\begin{array}{l}\text { UC in patients with PG (n } \\
=302) \\
n(\%)\end{array}$ & $\begin{array}{l}\text { UC in controls }(n= \\
1,497) \\
n(\%)\end{array}$ & OR $(95 \% \mathrm{Cl})$ & $\begin{array}{l}\mathrm{P} \\
\text { value }\end{array}$ \\
\hline All & 1,799 & $22(7.3 \%)$ & $8(0.5 \%)$ & $\begin{array}{l}14.62(6.45- \\
33.18)\end{array}$ & $\begin{array}{l}<.001 \\
0.0\end{array}$ \\
\hline \multicolumn{6}{|l|}{ Age, years } \\
\hline$<54$ & 841 & 15 (10.7\%) & $1(0.1 \%)$ & $\begin{array}{l}84.00(10.99- \\
641.61)^{-}\end{array}$ & $\begin{array}{l}< \\
0.001\end{array}$ \\
\hline$\geq 54$ & 958 & $7(4.3 \%)$ & $7(0.9 \%)$ & $\begin{array}{l}5.09(1.76- \\
14.72)\end{array}$ & 0.001 \\
\hline \multicolumn{6}{|l|}{ Gender } \\
\hline Male & 756 & $5(3.9 \%)$ & $5(0.8 \%)$ & $\begin{array}{l}5.12(1.46- \\
17.94)\end{array}$ & 0.005 \\
\hline Female & 1,043 & $17(9.7 \%)$ & $3(0.3 \%)$ & $\begin{array}{l}31.02(8.99- \\
107.10)\end{array}$ & $\hat{0}_{0.001}$ \\
\hline \multicolumn{6}{|l|}{ Ethnicity } \\
\hline Jews & 1,519 & $18(7.1 \%)$ & $7(0.6 \%)$ & $\begin{array}{l}13.63(5.63- \\
32.99)\end{array}$ & $\begin{array}{l}<.001 \\
0.0\end{array}$ \\
\hline Arabs & 280 & $4(8.5 \%)$ & $1(0.4 \%)$ & $\begin{array}{l}21.67(2.37- \\
198.63)\end{array}$ & $\begin{array}{l}<.001 \\
0.01\end{array}$ \\
\hline \multicolumn{6}{|c|}{ Smoking status } \\
\hline Smokers & 637 & $3(2.6 \%)$ & $3(0.6 \%)$ & $\begin{array}{l}4.63(0.92- \\
23.21)\end{array}$ & 0.041 \\
\hline $\begin{array}{l}\text { Non- } \\
\text { smokers }\end{array}$ & 1,162 & 19 (10.2\%) & $5(0.5 \%)$ & $\begin{array}{l}21.96(8.09- \\
59.62)\end{array}$ & $\hat{0.001}$ \\
\hline
\end{tabular}

We then conducted a sensitivity analysis following the exclusion of patients diagnosed with UC up to two years before PG. The association of UC and subsequent PG was attenuated in this analysis; still, it retained its statistical significance (OR, 9.88; 95\% Cl, 3.91-24.97).

Multivariate analyses were performed to adjust for putative confounding factors. The first model adjusted for $\mathrm{BMI}$ and smoking and found that the association between $\mathrm{PG}$ and $\mathrm{UC}$ remained independently significant $(\mathrm{OR}, 11.15 ; 95 \% \mathrm{Cl}, 4.73-26.25 ; P<0.001)$. The second model adjusted additionally for 
comorbidities and did not substantially alter the outcome (OR, 11.70; $95 \% \mathrm{Cl}, 4.90-27.93 ; P<0.001$; Table 3).

Table 3

The association between pyoderma gangrenosum and ulcerative colitis: Multivariate analyses*.

\begin{tabular}{|llll|}
\hline Variable & OR & 95\% Cl & P value \\
\hline Unadjusted model & 14.62 & $6.45-33.18$ & $\mathbf{0 . 0 0 1}$ \\
\hline First adjusted model ${ }^{1}$ & 11.15 & $4.73-26.25$ & $\mathbf{0 . 0 0 1}$ \\
\hline Second adjusted model ${ }^{2}$ & 11.70 & $4.90-27.94$ & $\mathbf{0 . 0 0 1}$ \\
\hline $\begin{array}{l}1 \\
\text { 2 adjusted for BMI and smoking. } \\
\text { abbreviations: Cl, confidence interval; } \\
\text { Bold:significant values }\end{array}$ & & \\
\hline
\end{tabular}

With respect to the temporal relationship between the two conditions, the mean (SD) latency between the diagnosis of UC and the development of PG was estimated at 7.09 (8.28) years, while the median (range) latency was 4.59 (0.00-28.72) years. Of interest, seven (31.8\%) patients with UC presented with PG in the initial year after their gastrointestinal diagnosis, whereas 11 (50.0\%) developed PG following the fifth year of their disease.

We then addressed the epidemiological features of patients with coexistent PG and UC $(n=22)$ as compared to the remaining patients with $P G(n=280)$. Patients with a dual diagnosis of $P G$ and UC were significantly younger at the onset of PG (45.1 [20.5] vs. 54.8 [20.6] years, respectively; $P=0.043)$, whereas the sex distribution and the ethnic background were comparable between the two subgroups (Table 4). Patients with both conditions had a lower prevalence of smoking ( $13.6 \%$ vs. $40.0 \%$, respectively; $P=0.001)$, while the mean BMI was comparable between the two subgroups (Table 4) 
Table 4

Comparison between patients with pyoderma gangrenosum and ulcerative colitis relative to the remaining patients with pyoderma gangrenosum

\begin{tabular}{|c|c|c|c|}
\hline Variable & OR & $95 \% \mathrm{Cl}$ & $P$ value \\
\hline Unadjusted model & 14.62 & $6.45-33.18$ & $<0.001$ \\
\hline First adjusted model ${ }^{1}$ & 11.15 & $4.73-26.25$ & $<0.001$ \\
\hline \multirow[t]{2}{*}{ Second adjusted model ${ }^{2}$} & 11.70 & $4.90-27.94$ & $<0.001$ \\
\hline & PG with UC $(n=22)$ & PG without UC $(n=280)$ & $P$ value \\
\hline Age at the onset of PG, years; mean \pm SD & $45.1+20.5$ & $54.8+20.6$ & 0.043 \\
\hline Male sex, $n(\%)$ & $5(22.7 \%)$ & $122(43.6 \%)$ & 0.056 \\
\hline Jewish ethnicity, n (\%) & $18(81.8 \%)$ & $237(84.6 \%)$ & 0.728 \\
\hline Body mass index; $\mathrm{Kg} / \mathrm{m} 2$, mean $\pm \mathrm{SD}$ & $26.7+3.9$ & $28.1+6.7$ & 0.335 \\
\hline Smokers, $\mathrm{n}(\%)$ & $3(13.6 \%)$ & $112(40.0 \%)$ & 0.014 \\
\hline
\end{tabular}

\section{Discussion}

The current case-control study discloses a 15 -folds increase in the odds of developing PG in patients with preceding UC. The association was proven stronger among females, young patients, and non-smokers. The median latency separating the diagnosis of $U C$ and the emergence of PG was 4.6 years. Relative to other patients with PG, patients with coexistent PG and UC were younger at the onset of the cutaneous manifestation and had a lower prevalence of smoking.

\section{Our knowledge of the investigated association based on the current literature}

The pooled incidence of PG was estimated in a recent meta-analysis summarizing 14 cohorts of 60,638 patients with IBD. PG developed in 0.1 to $2.6 \%$ of patients with IBD in different studies, providing a pooled estimate of $0.6 \%$. This data synthesis had additionally shown that the risk of PG was lower among patients with UC relative to those with Crohn`s disease (relative risk [RR], 0.84)[8].

IBD is the most frequently encountered underlying disease among patients with PG. A recent meta-analysis aimed to summarize the distribution and prevalence of underlying diseases among patients with PG. This study synthesized data across 21 cohorts, including 2,611 patients with PG, and found that the pooled prevalence of IBD was calculated at $17.6 \%(95 \% \mathrm{Cl}, 13.0-22.7)$, representing the leading single underlying condition. Of the aforementioned eligible studies, 17 distinguished between the subtypes of IBD and disclosed that the prevalence of UC ranged between $3.4 \%$ and $32.3 \%$ in different study populations, whilst its combined prevalence was calculated at $11.5 \%(95 \% \mathrm{Cl}, 7.2-16.6)[4]$. 


\section{Interpretations of the current study's main observations}

Despite the aforementioned data, the magnitude of the association between UC and the subsequent occurrence of PG is yet to be delineated. This gap in our knowledge rises from the lack of controlled studies comparing patients with PG and controls regarding the prevalence of preceding UC. Therefore, the current case-control study provides a novel epidemiological feature and conveys the important message that a foregoing diagnosis of UC is implicated with an 11- and 15 -folds increased multivariate and crude odds of having PG, respectively. In other words, individuals with UC have 11- to 15-fold greater odds to present with PG as compared to individuals without a diagnosis of UC. This conclusion is grounded upon the 'rare disease assumption' in case-control studies, theorizing that the $\mathrm{OR}$ approximates the RR in rare diseases[11]. Patients with UC should be aware of this substantial risk in order to minimize other risk factors of PG, including pathergy phenomenon derived from piercing, trauma, or unnecessary surgical procedures. Awareness of this risk may be of great benefit for physicians encountering rapidly evolving ulcers in patients with UC.

We found an explicit predilection for PG among female patients. Previous studies demonstrated that females were more predisposed to experience extraintestinal manifestations of IBD. In the meta-analysis of States et al.[8], female sex imposed a 32\% increased risk of developing PG among patients with IBD (RR, 1.33; $95 \% \mathrm{Cl} 1.16-1.52)$. In addition, Weizman et al.[12] noted a female preponderance (58\%) among their 80 patients with IBD-associated PG. In a Swedish population-based study including 1,274 patients with UC, Monsen et al.[13] observed a threefold increased odds of erythema nodosum among females. In their nationwide study of 950 Swiss patients with IBD, Vavricka et al.[14] found that extraintestinal manifestations were more prevalent among female patients with IBD (50\% vs. $34 \%$ in males). The aforementioned observations may imply for a putative role of estrogen, gene-hormone and/or gene-gene interactions in the induction pathogenesis of $P G$ and other extraintestinal UC manifestations of UC. Further research is required to spell out sex differences in PG.

Our study also denotes that patients with coexistent PG and UC were significantly younger at the onset of $P G$ relative to their remaining PG counterparts. The main interpretation of this finding stems from the fact that other patients with PG assumingly include a sizable proportion of patients with underlying hematological malignancies and rheumatoid arthritis who tend present at older ages, thus delaying the development of secondary PG. This finding aligns with previous studies tracking patients with IBDassociated PG, which reported younger ages of presentation[12, 15].

We revealed significantly greater odds of PG among patients of Arab ethnicity. The existence of ethnic predisposition in PG is still a debatable and inconclusive issue. While Nguyen et al.[16] did not find any ethnic predisposition for cutaneous manifestation in IBD, Farhi et al.[17] reported a higher risk of PG among IBD patients of an African race. Of note, both the incidence and prevalence rates of IBD were found lower among the Arab population residing in Israel relative to their Ashkenazi Jewish counterparts[18]. However, the Arab minority in Israel is typified by a higher burden of IBD when compared to Arab populations originating from other Middle-Eastern countries[19, 20]. In a study aiming to address morphological differences between IBD patients from different ethnic groups in Israel, patients of Arab ancestry were 
found to present more frequently with extraintestinal manifestations[18]. The latter finding lends credibility for the results of the current study.

The association between UC and PG was more protruding among non-smokers. This finding accords with the conclusions drawn from several observational studies suggesting that cigarette smoking does not confer risk for cutaneous manifestations in IBD (including both PG and erythema nodosum) [8, 21, 22]. In contrast, cigarette smoking was a significant predictor of PG in a cohort of patients with UC[23]. It is noteworthy that cigarette smoking emerged as a protective factor against the development of UC[24]. A meta-analysis summarizing 13 epidemiological studies has confirmed that current smoking imposes a protective effect in UC, with smokers having an OR of $0.58(95 \% \mathrm{Cl}, 0.45-0.75)$ to develop UC as compared to non-smokers[25]. Further research is required to better explicate the precise role of smoking in the development of cutaneous manifestation in UC.

\section{Strengths and limitations}

The case-control design enables the identification of the temporal relationship between diagnoses and allows the estimation of a causal relationship between the two conditions of interest[26]. The populationbased design and the recruitment of cases managed both in inpatient and outpatient settings diminish the likelihood of selection bias that may arise in other observational studies.

One of the main drawbacks interfering with the current study is the lack of data concerning the clinical characteristics and severity indices of the two diseases. Although the formal disease criteria were not directly required to recruit patients, the inclusion criteria were strict and valid; based on documentation by a certified dermatologist or dermatological wards for PG and on the chronic disease registry of CHS for UC. The latter was proven highly reliable[9].

\section{Conclusions}

In conclusion, the current population-based case-control study depicts that the presence of UC is associated with 15 -fold increased odds of having subsequent PG. The association was more protuberant among female, younger, and Arab patients, as well as among non-smokers. Estimates were not significantly altered in a sensitivity analysis excluding diagnoses up to 2 years before PG and in multivariate analysis adjusting for confounding factors. The diagnosis of UC preceded that of PG by a median interval of 4.6 years. Relative to other patients with PG, patients with concurrent PG and UC were younger at the onset of PG and had a lower prevalence of smokers. Prospective studies are necessary to reproduce our findings in other study populations. Physicians managing patients with UC should be aware of this increased risk of PG, and patients with UC should be advised to avoid additional triggering factors of PG.

\section{Methods}

\section{Study design and database}


We performed a retrospective population-based case-control study aiming to identify the risk of having subsequent PG following the diagnosis of UC.

The computerized dataset of Clalit Health Services (CHS) was the origin of the current study. CHS is the largest health care organization in Israel, providing healthcare services for approximately 4,400,000 enrollees. It provides a wide array of both public and private healthcare services. The computerized database of $\mathrm{CHS}$ is characterized by incessant real-time input from medical, administrative, and pharmaceutical operating systems. It was proven highly competent to enable the performance of epidemiological studies.

The chronic disease registry of CHS retrieves data from various sources, including from hospital and primary care reports, drug claims. These accumulated data is then manually cross-checked and validated by the primary care practitioner of each patient. This registry was considered as of vast reliability on the grounds of previous epidemiological studies[9].

The ethical committee of Ben-Gurion University approved the current study which was performed in accordance with declaration of Helsinki.

\section{Study population and covariates}

Between the years 2000 and 2018, we identified all individuals with diagnostic codes consistent with the diagnosis of PG. The inclusion of PG cases in this study was based on the following criteria: (i) a documented diagnosis of $P G$ at least twice in the medical file as registered by a community board-certified dermatologist, or (ii) documentation of a diagnosis of PG in hospital discharge letters from dermatological wards.

The diagnosis of UC was based on its registration in the chronic registry of CHS. To elaborate, it is based on the documentation of board-certified gastroenterologist, on the claims of UC-related drugs, and is eventually validated by the managing general practitioner.

The control group included up to 5 controls per patient, matched randomly by age, sex, and ethnicity. Age matching was based on the exact year of birth (1-year strata). The diagnosis date was used as an index date for the cases, and each matched control patient. Controls were ascertained to be alive and to contribute longitudinal data to the $\mathrm{CHS}$ dataset at their recruitment date.

Outcome measures were adjusted for comorbid underlying diseases utilizing the Charlson comorbidity index, a validated epidemiological method of measuring and categorizing comorbidities. This index has been demonstrated to be reliable in forecasting mortality[10]. Smoking status was classified as a current smoker or never/past smoker.

\section{Statistical analysis}

Baseline characteristics are described with means and standard deviations (SD)s for continuous variables, whereas percentages are used to represent categorical values. A comparison of the distribution of 
sociodemographic and clinical factors between patients with PG and controls was conducted using the Chi-square test and t-test, as indicated.

Logistic regression was used to calculate odds ratios (ORs) and 95\% Cls to compare cases and control with respect to the presence of UC. The association was calculated based on individuals who developed PG following the diagnosis of $\mathrm{UC}$ in accordance with the presence of a temporal relationship between exposure and outcome in case-control studies. Two-tailed P-values less than 0.05 were considered as statistically significant. All statistical analyses were performed using SPSS software, version 25 (SPSS, Armonk, NY: IBM Corp).

A sensitivity analysis was performed to ensure that the observed association was not overestimated due to ascertainment bias. This analysis was based on repeating all calculations with an exclusion period of two years prior to the diagnosis of PG. Thus, all individuals given a diagnosis of UC in this period were omitted from all calculations.

\section{List Of Abbreviations}

PG- pyoderma gangrenosum

UC- ulcerative colitis

IBD- inflammatory bowel disease

CHS- Clalit Healthcare Services

SD- standard deviation

OR- odds ratio

Cl- confidence interval

\section{Declarations}

\section{Ethics approval and consent to participate}

The current study was approved by the institutional ethical committee of Ben-Gurion University (0212-17$\mathrm{COM}$ ). Informed consent was deemed unnecessary according to local regulations since this is a retrospective non-interventional study.

\section{Consent for publication}

Not applicable 


\section{Competing interests}

None declared

\section{Funding}

Clinical Research Unit Pemphigoid Diseases (KFO 303) and Cluster of Excellence Precision Medicine in Chronic Inflammation (EXC 2167) both funded by Deutsche Forschungsgemeinschaft

\section{Authors' contributions}

KK and $A D C$ had full access to all of the data in the study and take responsibility for the integrity of the data and the accuracy of the data analysis. Study concept and design: KK. Acquisition, analysis, and interpretation of data: KK and RJK. Drafting of the manuscript: KK and GD Critical revision of the manuscript for important intellectual content: KK and DTB. Statistical analysis: DTB. Study supervision: ADC. All authors have read and approved the manuscript.

\section{Acknowledgements}

None

\section{Availability of data and materials-}

Not applicable

\section{References}

1. Marzano AV, Ishak RS, Saibeni S, Crosti C, Meroni PL, Cugno M. Autoinflammatory skin disorders in inflammatory bowel diseases, pyoderma gangrenosum and Sweet's syndrome: a comprehensive review and disease classification criteria. Clin. Rev. Allergy Immunol. [Internet]. 2013;45:202-10. Available from: http://www.ncbi.nlm.nih.gov/pubmed/23334898.

2. Marzano AV, Damiani G, Ceccherini I, Berti E, Gattorno M, Cugno M. Autoinflammation in pyoderma gangrenosum and its syndromic form (pyoderma gangrenosum, acne and suppurative hidradenitis). $\mathrm{Br}$ J Dermatol. 2017;176:1588-98.

3. Marzano AV, Borghi A, Meroni PL, Cugno M. Pyoderma gangrenosum and its syndromic forms: evidence for a link with autoinflammation. Br. J. Dermatol. 2016. p. 882-91.

4. Kridin K, Cohen AD, Amber KT. Underlying Systemic Diseases in Pyoderma Gangrenosum: A Systematic Review and Meta-Analysis. Am. J. Clin. Dermatol. [Internet]. 2018 [cited 2018 May 13]; Available from: http://www.ncbi.nlm.nih.gov/pubmed/29721816. 


\section{5. $10.1056 /$ NEJMra1102942}

Danese S, Fiocchi C. Ulcerative Colitis N, Engl. J. Med. [Internet]. Massachusetts Medical Society; 2011 [cited 2017 Jan 7];365:1713-25. Available from:

http://www.nejm.org/doi/abs/10.1056/NEJMra1102942.

6. Ng SC, Shi HY, Hamidi N, Underwood FE, Tang W, Benchimol El, et al. Worldwide incidence and prevalence of inflammatory bowel disease in the 21 st century: a systematic review of populationbased studies. Lancet. 2017.

7. Ott C, Scholmerich J. Extraintestinal manifestations and complications in IBD. Nat. Rev. Gastroenterol. Hepatol. 2013.

8. States V, O’Brien S, Rai JP, Roberts HL, Paas M, Feagins K, et al. Pyoderma Gangrenosum in Inflammatory Bowel Disease: A Systematic Review and Meta-Analysis. Dig. Dis. Sci. 2020.

9. Rennert G, Peterburg Y. Prevalence of selected chronic diseases in Israel. Isr. Med. Assoc. J. [Internet]. 2001 [cited 2017 Jan 30];3:404-8. Available from: http://www.ncbi.nlm.nih.gov/pubmed/11433630.

10. Charlson ME, Pompei P, Ales KL, MacKenzie CR. A new method of classifying prognostic comorbidity in longitudinal studies: development and validation. J. Chronic Dis. [Internet]. 1987;40:373-83. Available from: http://www.ncbi.nlm.nih.gov/pubmed/3558716.

11. Greenland S, Thomas DC. On the need for the rare disease assumption in case-control studies. Am. J. Epidemiol. 1982.

12. Weizman AV, Huang B, Targan S, Dubinsky M, Fleshner P, Kaur M, et al. Pyoderma gangrenosum among patients with inflammatory bowel disease: A descriptive cohort study. J. Cutan. Med. Surg. 2015.

13. Monsen U, Sorstad J, Hellers G, Johansson C. Extracolonic Diagnoses in Ulcerative Colitis: An Epidemiological Study. Am. J. Gastroenterol. 1990.

14. Vavricka SR, Brun L, Ballabeni P, Pittet V, Prinz Vavricka BM, Zeitz J, et al. Frequency and risk factors for extraintestinal manifestations in the swiss inflammatory bowel disease cohort. Am. J. Gastroenterol. 2011.

15. Polcz M, Gu J, Florin T. Pyoderma gangrenosum in inflammatory bowel disease: The experience at Mater Health Services' Adult Hospital 1998-2009. J. Crohn's Colitis. 2011.

16. Nguyen GC, Torres EA, Regueiro M, Bromfield G, Bitton A, Stempak J, et al. Inflammatory bowel disease characteristics among African Americans, Hispanics, and non-Hispanic whites: Characterization of a large North American cohort. Am. J. Gastroenterol. 2006.

17. Farhi D, Cosnes J, Zizi N, Chosidow O, Seksik P, Beaugerie L, et al. Significance of erythema nodosum and pyoderma gangrenosum in inflammatory bowel diseases: A cohort study of 2402 patients. Medicine (Baltimore). 2008.

18. Mahamid M, Bar-Gil Shitrit A, Amara H, Koslowsky B, Ghantous R, Safadi R. Ethnic Variations in Inflammatory Bowel Diseases Among Israel's Populations. Isr. Med. Assoc. J. 2019.

19. Malaty HM, Hou JK, Thirumurthi S. Epidemiology of inflammatory bowel disease among an indigent multi-ethnic population in the United States. Clin. Exp. Gastroenterol. 2010. 
20. Finlay DG, Basu D, Sellin JH. Effect of race and ethnicity on perceptions of inflammatory bowel disease. Inflamm. Bowel Dis. 2006.

21. Roberts H, Rai SN, Pan J, Rao JM, Keskey RC, Kanaan Z, et al. Extraintestinal manifestations of inflammatory bowel disease and the influence of smoking. Digestion. 2014.

22. Karmiris K, Avgerinos A, Tavernaraki A, Zeglinas C, Karatzas P, Koukouratos T, et al. Prevalence and Characteristics of Extra-intestinal Manifestations in a Large Cohort of Greek Patients with Inflammatory Bowel Disease. J. Crohn's Colitis. 2016.

23. Manguso F, Sanges M, Staiano T, Gargiulo S, Nastro P, Gargano D, et al. Cigarette smoking and appendectomy are risk factors for extraintestinal manifestations in ulcerative colitis. Am. J. Gastroenterol. [Internet]. United States; 2004;99:327-34. Available from: http://ovidsp.ovid.com/ovidweb.cgi?T=JS\&PAGE=reference\&D=med5\&NEWS=N\&AN=15046225.

24. $10.1007 / \mathrm{s} 12026-018-9021-8$

Kridin K, Zamir H, Cohen AD. Cigarette smoking associates inversely with a cluster of two autoimmune diseases: ulcerative colitis and pemphigus. Immunol. Res. [Internet]. 2018 [cited 2019 Feb 18];66:5556. Available from: http://link.springer.com/10.1007/s12026-018-9021-8.

25. Mahid SS, Minor KS, Soto RE, Hornung CA, Galandiuk S. Smoking and inflammatory bowel disease: a meta-analysis. Mayo Clin. Proc. [Internet]. 2006;81:1462-71. Available from: http://www.ncbi.nlm.nih.gov/pubmed/7120402\%5Cnhttp://www.ncbi.nlm.nih.gov/pubmed/17120402.

26. Höfler M. The Bradford Hill considerations on causality: a counterfactual perspective. Emerg. Themes Epidemiol. [Internet]. 2005;2:11. Available from: http://www.pubmedcentral.nih.gov/articlerender.fcgi? artid $=1291382 \&$ tool=pmcentrez\&rendertype $=$ abstract. 